\title{
USO DE MEDICAMENTOS EN ADULTOS MAYORES DE BOGOTÁ, COLOMBIA
}

\author{
Carlos Cano-Guitierrez ${ }^{1, a}$, Rafael Samper-Ternent ${ }^{1, b}$, Javier Cabrera $^{1, \mathrm{~b}}$, Diego Rosselli1,c
}

\begin{abstract}
RESUMEN
Objetivos. Determinar la frecuencia y tipo de medicamentos que utiliza un grupo de adultos mayores que viven en la capital colombiana, y cuáles son potencialmente inapropiados, con base en los criterios revisados de Beers. Materiales $y$ métodos. Los datos fueron obtenidos del estudio SABE (estudio de salud, bienestar y envejecimiento) que se llevó a cabo en áreas urbanas y rurales de Bogotá en el 2012. Se utilizó la prueba U de Mann-Whitney para analizar las diferencias por sexo en los diferentes grupo de edad con relación al número de medicamentos tomados. Resultados. Un total de 1268 $(63,4 \%)$ de los adultos mayores fueron mujeres; entre los participantes, $1514(75,7 \%)$ tenían 5 años de educación básica o menos. El promedio de medicamentos tomados en general fue de 3,24 siendo mayor en mujeres $(3,50)$ que en hombres $(2,79)$. Un total de 549 sujetos $(27,4 \%)$ reportaron recibir cinco medicamentos o más. De los medicamentos utilizados, $443(6,9 \%)$ eran potencialmente inapropiados de acuerdo con los criterios de Beers. La prevalencia autorreportada de hipertensión fue de $56,9 \%$ y para diabetes de $17,5 \%$. Solo 311 sujetos (15,6\%) no tomaban ningún medicamento. Conclusiones. El uso de medicamentos inapropiados es un problema importante que afecta a los adultos mayores en Bogotá. Estudios adicionales deberán identificar factores que aumentan el riesgo de polifarmacia y uso inapropiado de medicamentos y diseñar intervenciones para prevenir el uso inapropiado de medicamentos entre adultos mayores.
\end{abstract}

Palabras clave: Polifarmacia; Adultos mayores; Colombia (fuente: DeCS BIREME).

\section{MEDICATION USE AMONG OLDER ADULTS IN BOGOTA, COLOMBIA}

\begin{abstract}
Objectives. To determine the frequency and types of medication used by older adults living in the Colombian capital, Bogota, and to identify the use of potentially inappropriate medications according to the revised Beers criteria. Materials and Methods. Data were obtained from the health, welfare, and aging (SABE, Spanish: salud, bienestar y envejecimiento) study conducted in both urban and rural areas of Bogota in 2012. The Mann-Whitney U test was used to analyze sexrelated differences in the number of medications used among age-stratified subgroups. Results. Of 2000 subjects, 1,268 $(63.4 \%)$ were women; furthermore, $1,514(75.7 \%)$ of the total participants had $\leq 5$ years of basic education. The self-reported prevalence rates of hypertension and diabetes were $56.9 \%$ and $17.5 \%$, respectively. The average number of medications per subject was 3.24, and it was higher in women (3.50) than in men (2.79). A total of 549 subjects $(27.4 \%)$ reported using $\geq 5$ medications. Only 311 subjects (15.6\%) reported not using any medication. According to the Beers criteria, 443 $(6.9 \%)$ of the medications were potentially inappropriate. Conclusions. The use of inappropriate medications is a major problem among older adults in Bogota. Further studies should identify factors that increase the risks of polypharmacy and inappropriate medication use and design interventions that would allow older adults to avoid these risks.
\end{abstract}

Key words: Polypharmacy; Older adults; Colombia (source: MeSH NLM).

\section{INTRODUCCIÓN}

Debido al acelerado envejecimiento poblacional, el problema de la polifarmacia entre adultos mayores se ha convertido en una preocupación cada vez mayor para los sistemas de salud y para la población en general. La mayoría de los estudios sobre el uso apropiado de medicamentos, y sobre estrategias para prevenir y gestionar la polifarmacia, viene de países desarrollados (1). Sin embargo, la polifarmacia es cada vez más reconocida como problema importante de salud en países en vías de desarrollo, donde un menor control en la prescripción y entrega de medicamentos es frecuente. Es evidente que los adultos mayores son el grupo de población que consume el mayor número de

\footnotetext{
Pontificia Universidad Javeriana. Bogotá, Colombia

Médico geriatra; ${ }^{\mathrm{b}}$ médico residente de geriatría; ${ }^{\mathrm{c}}$ médico neurólogo

Recibido: 23/02/2016 Aprobado: 06/07/2016
}

Citar como: Cano-Guitierrez C, Samper-Ternent R, Cabrera J, Rosselli D. Uso de medicamentos en adultos mayores de Bogotá, Colombia. Rev Peru Med Exp Salud Publica. 2016;33(3):419-24. doi: 10.17843/rpmesp.2016.333.2292 
medicamentos, y que existe una falta de supervisión en el uso de los mismos. Estas condiciones resultan en un aumento en el riesgo de hospitalización, disminución en la capacidad funcional y, en muchos casos, la muerte. Sin embargo, algunos autores han identificado limitaciones metodológicas en la manera en que se desarrollan y establecen los criterios clínicos sobre la prescripción apropiada para adultos mayores ${ }^{(2)}$. En Latinoamérica, la mayor parte de los estudios viene de Brasil ${ }^{(3,4)}$ y México ${ }^{(5)}$. Solo existe un estudio sobre el uso de medicamentos en la población de adultos mayores en Colombia y los datos provienen de un solo hospital ${ }^{(6)}$.

En el estudio SABE (acrónimo que significa estudio de salud, bienestar y envejecimiento) realizado en Brasil (4) en 1115 adultos de 65 años o más, la polifarmacia fue definida como el uso de cinco o más medicamentos, encontrando una prevalencia del $36 \%$ donde el sexo femenino, ser mayor de 75 años, ingresos económicos más altos, ser activo desde el punto de vista laboral, peor autorreporte de salud, diagnóstico de hipertensión, diabetes, enfermedades reumáticas y problemas cardiacos, fueron asociados con una mayor prevalencia de polifarmacia. Por el contrario, una mayor independencia, medida según la capacidad para usar transporte público, se asoció con una menor prevalencia. Los medicamentos para los sistemas cardiovascular, gastrointestinal y endocrino fueron los más utilizados (4). Otro estudio realizado en la ciudad brasileña de Ribeirão Preto, en 1000 adultos mayores que utilizaron una farmacia ambulatoria ${ }^{(3)}$ donde la polifarmacia fue definida como el uso de más de cinco medicamentos la prevalencia hallada fue de $60,1 \%$, se observó además un alto uso de medicamentos potencialmente inapropiados $(59,2 \%)$ según los criterios de Beers actualizados en 2012 (3) donde el sexo femenino, el uso de medicamentos de venta libre y el uso de medicamentos psicotrópicos, dio como resultado un riesgo incrementado para el uso de medicamentos potencialmente inapropiado.

Otro estudio, que utilizó datos de México ${ }^{(5)}$, definió la polifarmacia como el uso de 3 o más medicamentos. Un total de 73 adultos de 65 años o más fue entrevistado; la polifarmacia fue más prevalente en mujeres y en el grupo de edad de 75 a 79 años. Los medicamentos más utilizados fueron el ácido acetilsalicílico (17\%), paracetamol (15\%), naproxeno (13\%), captopril $(11 \%)$ y ranitidina $(10 \%)$.

El estudio realizado en Colombia en 2007, en las unidades de consulta externa y urgencias de un hospital en Bogotá (6), los autores hallaron que el número promedio de medicamentos por persona era de 3,2. Este estudio utilizó los criterios de Beers de 2002 y los autores reportaron que $20,7 \%$ de los pacientes recibió medicamentos potencialmente inapropiados.
Los individuos con enfermedades cardiovasculares y neurológicas tenían una mayor posibilidad de recibir medicamentos potencialmente inapropiados.

El objetivo del presente estudio es determinar la frecuencia y tipo de medicamentos que utiliza un grupo de adultos mayores que viven en la capital colombiana, y cuáles son potencialmente inapropiados, con base en los criterios revisados de Beers.

\section{MATERIALES Y MÉTODOS}

Se realizó un estudio de tipo transversal llevado a cabo en 2012 tanto en áreas rurales como urbanas de Bogotá utilizando datos de un estudio poblacional que se realizó siguiendo la metodología de un estudio multinacional poblacional llevado a cabo en siete ciudades capitales de Latinoamérica (Bridgetown, Buenos Aires, Ciudad de México, La Habana, Montevideo, Santiago de Chile y Sao Paulo). El estudio original (SABE) fue diseñado por la Organización Panamericana de la Salud y varios centros académicos en los Estados Unidos para determinar los estándares de vida y condiciones relacionadas con la salud de los adultos mayores ${ }^{(7)}$. En 2012 utilizamos este cuestionario modificado y actualizado para entrevistar a adultos mayores en Bogotá. También utilizamos los criterios de Beers recientemente actualizados para identificar medicamentos inapropiados en adultos mayores ${ }^{(8)}$. Los criterios de Beers fueron originalmente publicados en 1991 y se enfocaron en adultos mayores institucionalizados y las actualizaciones subsiguientes en 1997,2003 y $2012^{(9,10)}$.

El estudio SABE incluye once temas principales: 1) identificación de adultos mayores a ser entrevistados y residencia de los mismos; 2) características de vivienda; 3) datos personales y familiares; 4) experiencias a lo largo de la vida de violencia; 5) estado cognitivo; 6) estado de salud; 7) caracterización y causas de discapacidades; 8) evaluación funcional; 9) uso de medicamentos y acceso a servicios de salud; 10) evaluación antropométrica; 11) historia de salud, enfermedad e historia social (componente autobiográfico). Este artículo se enfoca en el uso de medicamentos.

La muestra se recolectó por conglomerados en múltiples etapas (sectores, subsecciones de barrios, bloques y grupos de diez casas), diseñada para obtener una muestra representativa mediante la ponderación de las principales variables sociodemográficas en la selección de cada conglomerado. Los cálculos originales del tamaño de la muestra estimaron un tamaño de 1685 sujetos; asumiendo una tasa de rechazo del $45 \%$ (con base en reportes anteriores del estudio SABE donde la tasa de rechazo fue de $40 \%$ ), la muestra ajustada se incrementó a 2444 sujetos. De este grupo, 2000 adultos aceptaron entrar al estudio y fueron entrevistados (una 
tasa de aceptación de $81,4 \%$ ). Un equipo compuesto por un supervisor, 3-4 entrevistadores y un profesional especializado en antropometría llevó a cabo el estudio puerta a puerta, que tomó alrededor de $2 \mathrm{~h}$ por cada sujeto entrevistado.

Los resultados del estudio fueron recolectados en papel y luego fueron transferidos a una base de datos de manera independiente por dos programadores. Para el análisis descriptivo se estimaron promedios, errores estándares y porcentajes. Para las diferencias por sexo en cada grupo de edad con relación al número de medicamentos tomados, utilizamos la prueba $U$ de Mann-Whitney. La correlación entre la edad y el número de medicamentos tomados se evaluó utilizando la prueba de correlación de Spearman-Rank.

Todos los participantes y sus cuidadores firmaron un consentimiento informado antes de la entrevista. El estudio se realizó cumpliendo con los estándares éticos colombianos (Resolución 8430 de 1993) y fue aprobado por el Comité de Ética de la Facultad de Medicina de la Pontifica Universidad Javeriana.

\section{RESULTADOS}

Un total de 2000 sujetos de más de 60 años respondió al cuestionario. En la Tabla 1 se observa las características generales de la muestra. Los adultos mayores de 80 años de edad fueron el $17 \%$ de la muestra total; más de $70 \%$ de los encuestados tiene 5 años de educación o menos. Las enfermedades de los participantes fueron autorreportadas, y se listan en la Tabla 2. Hipertensión, artritis y osteoporosis fueron las condiciones más prevalentes con mayor frecuencia en mujeres.

Tabla 1. Características de la población de estudio $(n=2000)$

\begin{tabular}{|c|c|}
\hline Variable & n (\%) \\
\hline Grupos de edad* & $71,2(8,1)$ \\
\hline $60-69$ & $960(48,0)$ \\
\hline $70-79$ & $702(35,0)$ \\
\hline $80-89$ & $301(15,1)$ \\
\hline$\geq 90$ & $37(1,9)$ \\
\hline \multicolumn{2}{|l|}{ Sexo } \\
\hline Mujeres & $1268(63,4)$ \\
\hline Hombres & $732(36,5)$ \\
\hline \multicolumn{2}{|c|}{ Educación en años } \\
\hline Ninguna & $235(11,7)$ \\
\hline $1-5$ & $1279(64,0)$ \\
\hline $6-11$ & $483(24,1)$ \\
\hline 12 o más & $3(0,2)$ \\
\hline
\end{tabular}

*Promedio (desviación estándar)
Tabla 2. Autorreporte de condiciones médicas $(n=2000)$

\begin{tabular}{|c|c|c|}
\hline & Hombres & Mujeres \\
\hline & n (\%) & n (\%) \\
\hline Hipertensión & $416(55,4)$ & $749(60,0)$ \\
\hline Artritis & $138(18,4)$ & $495(39,6)$ \\
\hline $\begin{array}{l}\text { Osteoporosis u } \\
\text { osteopenia }\end{array}$ & $64(8,5)$ & $425(34,0)$ \\
\hline Diabetes & $131(17,4)$ & $218(17,5)$ \\
\hline $\begin{array}{l}\text { Enfermedad pulmonar } \\
\text { obstructiva crónica }\end{array}$ & $115(15,3)$ & $219(17,5)$ \\
\hline Cáncer & $50(6,7)$ & $73(5,8)$ \\
\hline $\begin{array}{l}\text { Insuficiencia cardíaca } \\
\text { congestiva }\end{array}$ & $49(6,5)$ & $80(6,4)$ \\
\hline
\end{tabular}

Se evaluó el autorreporte de salud; $122(6,1 \%)$ de los encuestados calificaron su salud como "excelente"; 821 $(41,1 \%)$ como muy buena; 911 (45,6\%) como "regular", y $144(6,2 \%)$ como "mala". También se evaluó la cognición con una versión modificada del mini-mental test (MMSE) (10) donde una calificación menor a 11 indicaba una entrevista por proxi. Un puntaje menor a 13 de 19 se usó como punto de corte para considerar deterioro cognitivo; un total de 252 encuestados (12,6\%) tuvo una calificación menor a 13/19.

Se indicó a los participantes que mostraran al entrevistador todos los medicamentos que estaban tomando, y el entrevistador registró el nombre genérico, la dosis y la frecuencia en el uso de cada medicamento. Algunos hallazgos importantes incluyen: 1065 (90,8\%) de los 1173 sujetos que declararon tener hipertensión estaban tomando medicamentos, mientras que $720 \quad(61,4 \%)$ estaban "siguiendo una dieta". De los 358 participantes con diabetes, $93(26,0 \%)$ estaba recibiendo insulina.

La Tabla 3 muestra los medicamentos más utilizados entre los encuestados. Agrupamos los medicamentos por categorías en la Tabla 4. Los 2000 encuestados recibieron un total de 6470 medicamentos diferentes, para un promedio de 3,24 medicamentos por persona. La mayor parte de los medicamentos $(5933,91,7 \%)$ fueron prescrito por un médico, $174(2,7 \%)$ por un pariente y $140(2,2 \%)$ fueron autoprescritos. De la muestra total, 1689 sujetos $(84,4 \%)$ estaban recibiendo por lo menos un medicamento, mientras que $548(27,4 \%)$ recibían cinco medicamentos o más.

Según los criterios de Beers actualizados se encontraron un total de 33 medicamentos potencialmente inapropiados usados, la Tabla 5 presenta una lista de los cinco más frecuentemente usados. Un total de $6,9 \%$ de los medicamentos utilizados cumplen con los 
Tabla 3. Medicamentos más consumidos por los adultos mayores

\begin{tabular}{lc}
\hline & $\mathbf{n = 1 6 8 9 ( \% )}$ \\
\hline Acido acetilsalicilico & $594(35,2)$ \\
\hline Losartan & $495(29,3)$ \\
Omeprazol & $399(23,6)$ \\
\hline Acetaminofen & $372(22,0)$ \\
Enalapril & $331(19,6)$ \\
Levotiroxina & $278(16,5)$ \\
Hidroclorotiazida & $272(16,1)$ \\
Lovastatina & $257(15,2)$ \\
Calcio & $209(12,4)$ \\
Metoprolol & $184(10,9)$ \\
\hline
\end{tabular}

criterios de Beers y son potencialmente inapropiados para adultos mayores.

La Tabla 6 muestra la asociación entre sexo, edad y número de medicamentos recibidos encontrando una correlación significativa, entre la menor edad y el número de medicamentos tomados así como una diferencia en el total de medicamentos consumidos según sexo.

\section{DISCUSIÓN}

Reducir la polifarmacia y evitar el consumo de medicamentos inapropiados es un objetivo primordial en el cuidado de personas mayores (11). La polifarmacia aumenta el riesgo de interacciones medicamentomedicamento y medicamento-enfermedad (11). Una de las definiciones más comúnmente utilizada es el

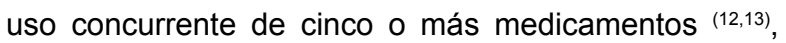
definición que ha sido utilizada en otros estudios latinoamericanos ${ }^{(4,14)}$. Sin embargo, es importante resaltar que hay individuos que requieren más de cinco medicamentos para controlar múltiples condiciones médicas. La polifarmacia es perjudicial por diferentes razones ${ }^{(15)}$, primero, las interacciones medicamentomedicamento se convierten en un problema importante que requiere supervisión cercana ${ }^{(16)}$, segundo, los estudios han mostrado que mientras más medicamentos

Tabla 4. Categorías de fármacos más consumidos por los adultos mayores

\begin{tabular}{lr}
\hline & $\mathbf{n = 1 6 8 9 ( \% )}$ \\
\hline Antihipertensivos & $1479(87,6)$ \\
\hline Antiagregantes plaquetarios & $615(36,4)$ \\
Inhibidores de la bomba de protones & $417(24,7)$ \\
Diuréticos & $415(24,6)$ \\
Estatinas & $337(20,0)$ \\
\hline Hipoglicemiantes & $298(17,6)$ \\
Hormonas tiroideas & $278(16,5)$ \\
Vitaminas & $198(11,7)$ \\
Antiinflamatorios no esteroideos & $161(9,5)$ \\
\hline Antidepresivos & $76(4,5)$ \\
\hline
\end{tabular}

Tabla 5. Ingredientes activos potecialmente inapropiados más consumidos por los adultos mayores

\begin{tabular}{lc}
\hline & $\mathbf{n = 1 6 8 9 ( \% )}$ \\
\hline Diclofenaco & $50(3,0)$ \\
Naproxeno & $50(3,0)$ \\
Nifedipina & $47(2,8)$ \\
Ibuprofeno & $47(2,8)$ \\
Espironolactona & $39(2,3)$ \\
\hline
\end{tabular}

se utilicen, es menor la adherencia a los tratamientos médicos ${ }^{(17,18)}$. Finalmente, la calidad de vida decrece de manera significativa a medida que el número de medicamentos se incrementa ${ }^{(18)}$.

El aumento en la polifarmacia está impulsado por el crecimiento de la población adulta mayor y la alta prevalencia de la multimorbilidad. La polifarmacia ha sido asociada con la presencia de múltiples enfermedades, edad avanzada, ser mujer, bajo nivel de salud autorreportada, menores ingresos, baja educación, vivir en una institución y número de visitas médicas por año (13). El conocimiento de los pacientes sobre su tratamiento y su salud y las expectativas individuales pueden cumplir un papel importante, por ende, los pacientes con mayor educación pueden tener mejor acceso a información sobre medicamentos y pueden tener una comunicación médico-paciente más activa e influenciar en la prescripción de una nueva droga ${ }^{(13)}$. Sin embargo, poca educación también puede influenciar la presencia de polifarmacia; el bajo nivel educativo de nuestra muestra podría coincidir con este hallazgo. Incorporar listas de verificación para identificar la prescripción inadecuada de medicamentos entre adultos mayores durante chequeos rutinarios, entre otras intervenciones descritas, pueden ser útiles para disminuir la polifarmacia. Es importante también identificar la indicación de cada medicamento, los riesgos de reacciones adversas, la capacidad del paciente o del cuidador de administrar los medicamentos de manera segura, así como las intervenciones educativas a los cuidadores, como medidas orientadas a reducir las hospitalizaciones y muertes relacionadas con medicamentos ${ }^{\left({ }^{19}\right)}$. Nuestros datos muestran una alta tasa de uso de medicamentos potencialmente inapropiados o con escasa evidencia en cuanto a su efectividad. Un ejemplo es la glucosamina.El $4,2 \%$ de nuestra publicación tomaba este medicamento.Un reciente metaanálisis muestra que, en comparación con el uso de placebo, hay pocos beneficios de la glucosamina en el manejo de distintos tipos de osteoartritis ${ }^{(21)}$. Esta información apoya la noción de que el bajo nivel educativo, la multimorbilidad y la falta de control por parte de los médicos, al igual que la falta de información actualizada, pueden incrementar el riesgo de polifarmacia y ponen en peligro el bienestar del adulto mayor. 
Tabla 6. Número de medicamentos consumidos según sexo y grupos de edad

\begin{tabular}{|c|c|c|c|c|c|c|c|}
\hline \multirow[b]{2}{*}{ Edad (años) } & \multicolumn{3}{|c|}{ Hombres } & \multicolumn{3}{|c|}{ Mujeres } & \multirow[b]{2}{*}{ Valor $p^{*}$} \\
\hline & $\mathbf{n}$ & Media (D.E.) & Mediana (IQR) & $\mathbf{n}$ & Media & Mediana (IQR) & \\
\hline $60-64$ & 185 & $2,1(2,35)$ & $1(0,34)$ & 321 & $2,93(2,45)$ & $3(0,32)$ & $<0,001$ \\
\hline $65-69$ & 164 & $2,35(2,06)$ & $2(0,32)$ & 290 & $3,14(2,56)$ & $3(0,29)$ & $<0,01$ \\
\hline $70-74$ & 151 & $3,05(2,46)$ & $3(0,33)$ & 247 & $3,81(2,50)$ & $4(0,22)$ & $<0,01$ \\
\hline $75-79$ & 124 & $3,34(2,74)$ & $3(0,30)$ & 180 & $3,91(2,75)$ & $4(0,27)$ & $<0,05$ \\
\hline $80-84$ & 83 & $3,43(3,03)$ & $3(0,33)$ & 123 & $4,46(2,71)$ & $4(0,19)$ & $<0,01$ \\
\hline $85-89$ & 33 & $3,33(2,82)$ & $2(0,50)$ & 62 & $3,87(2,65)$ & $3,5(0,21)$ & $>0,5$ \\
\hline 90 a más & 11 & $4,82(3,28)$ & $4(0,06)$ & 26 & $3,50(2,47)$ & $4(0,30)$ & $>0,5$ \\
\hline Total & 751 & $2,79(2,56)$ & $2(0,33)$ & 1249 & $3,50(2,61)$ & $3(0,27)$ & $<0,001$ \\
\hline
\end{tabular}

D.E: desviación estándar. IQR rango intercuartil. *U de Mann-Whitney

En general, la frecuencia de polifarmacia en nuestro estudio es menor que la que otros estudios han reportado. Gnijdiv et al. ${ }^{(12)}$, en Australia, encontraron un promedio de 4,0 medicamentos por individuo en su muestra de 1705 adultos mayores de 70 años o más. El estudio SABE Sao Paulo encontró que $36 \%$ de los adultos mayores tomaban cinco medicamentos o más, en comparación con nuestro 27\%; y los adultos mayores en dicho estudio tomaban un promedio de 3,6 medicamentos por individuo, en comparación con nuestros 3,2 (4). También encontraron una mayor frecuencia de polifarmacia en mujeres que en hombres, un hecho que ha sido descrito en otros estudios ${ }^{(13,21)}$. Las frecuencias de polifarmacia son aun más altas en estudios basados en hospitales: Marzi et al. (22) encontraron 6,1 medicamentos por individuo en una población de 179 adultos mayores en un hospital argentino. La proporción de medicamentos potencialmente inapropiados de acuerdo con los criterios de Beers también fue mayor: de las 1086 prescripciones, se encontró que $179(16,5 \%)$ eran potencialmente inapropiadas. Los números de un estudio basado en la comunidad en Taiwán ${ }^{(21)}$ también son mayores que los nuestros (19,1\% utilizando los criterios de Beers). La comparación de frecuencias de polifarmacia, por ende, debe tomar en cuenta el entorno del estudio: los estudios basados en comunidades muestran figuras más bajas que las de adultos mayores institucionalizados ${ }^{(23,24)}$ y los estudios basados en hospitales ${ }^{(22)}$, debido a que el entorno puede tener sesgos de selección por una muestra menos sana.

A pesar de la diferencia en las prevalencias que podrían derivarse de una variedad de fuentes como la recolección de los datos, el tipo de población analizado, y el sesgo de recuerdo que depende del tipo de estudio, hay grupos de medicamentos que se ha demostrado afectan a los adultos mayores y que deberían evitarse o usarse con extremo cuidado. Por ejemplo, se ha establecido que las drogas sedantes-hipnóticas (por ejemplo benzodiacepinas) incrementan el riesgo de caídas (21) y el deterioro cognitivo ${ }^{(23)}$. También se ha mostrado que hay un mayor riesgo de eventos adversos por medicamentos (por ejemplo hemorragias gastrointestinales) entre pacientes mayores que toman drogas antiinflamatorias no esteroideas ${ }^{(25)}$. En nuestro estudio estos medicamentos estuvieron entre los más prescritos.

En conclusión, la polifarmacia es un problema importante que afecta a los adultos mayores en Bogota, Colombia. En este estudio, los participantes recibieron en promedio tres medicamentos. Las categorías más comunes fueron los medicamentos antihipertensivos, seguidos de los medicamentos antiplaquetarios y los inhibidores de bomba de protones. De acuerdo con los criterios de Beers, casi $7 \%$ de los medicamentos recibidos por los participantes de nuestro estudio son potencialmente inapropiados. Los avances en el conocimiento y la tecnología médica han prolongado la vida, y esto ha tenido como resultado que muchos individuos vivan más tiempo con condiciones crónicas que requieren medicamentos por periodos mayores, incrementando así el riesgo de prescripciones potencialmente inapropiadas. Todos los profesionales de la salud involucrados en el cuidado de adultos mayores en Colombia deben llevar a cabo una evaluación de rutina de los medicamentos. Estos resultados deben informar a los formuladores de políticas y proveedores de cuidado de la salud sobre la importancia de identificar medicamentos que son potencialmente perjudiciales para los adultos mayores, revisando constantemente las prácticas de prescripción para evitar eventos que ponen en peligro la vida y mejorar el cuidado general de este grupo poblacional de rápido crecimiento.

Contribuciones de los autores: CCG conceptualizó el estudio y fue el director del proyecto; RST y JC proporcionaron apoyo logístico para la recolección de información y manejo de los datos; DR hizo el análisis estadístico y escribió la primera versión del manuscrito, todos los autores participaron en el análisis y la revisión de la versión final del manuscrito.

Fuentes de financiamiento: este estudio fue financiado por COLCIENCIAS, proyecto \# 120354531692 y por la Pontificia Universidad Javeriana.

Conflictos de interés: los autores declaran no tener conflictos de interés en la publicación de este artículo. 


\section{REFERENCIAS BIBLIOGRÁFICAS}

1. Hajjar ER, Cafiero AC, Hanlon JT. Polypharmacy in elderly patients. Am J Geriatr Pharmacother. 2007;5(4):345-51. doi: 10.1016/j. amjopharm.2007.12.002.

2. Marriott J, Stehlik P. A critical analysis of the methods used to develop explicit clinical criteria for use in older people. Age Ageing. 2012;41(4):441-50. doi: 10.1093/ageing/afs064.

3. Baldoni Ade O, Ayres LR, Martinez EZ, Dewulf Nde L, Dos Santos V, Pereira LR. Factors associated with potentially inappropriate medications use by the elderly according to Beers criteria 2003 and 2012. Int J Clin Pharm. 2014;36(2):316-24. doi: 10.1007/s11096-013-9880-y.

4. Carvalho MF, Romano-Lieber NS, Bergsten-Mendes G, Secoli SR, Ribeiro E, Lebrão ML, et al. Polypharmacy among the elderly in the city of São Paulo, Brazil - SABE Study. Rev Bras Epidemiol. 2012;15(4):817-27.

5. Sánchez-Gutiérrez R, Flores-García A, Aguilar-Garcia P, Ruiz-Bernes $S$, Sanchez-Beltran CA, BenitezGuerrero V, et al. Efectos de la Polifarmacia sobre la calidad de vida en adultos mayores. Revista Fuente. 2012;10:70-5.

6. Holguín-Hernández E, OrozcoDíaz JG. Medicación potencialmente inapropiada en ancianos en un hospital de primer nivel, Bogotá 2007. Rev Salud Publica (Bogota). 2010;12(2):287-99.

7. Menéndez J, Guevara A, Arcia N, León Díaz EM, Marín C, Alfonso JC. Enfermedades crónicas y limitación funcional en adultos mayores: estudio comparativo en siete ciudades de America Latina y el Caribe. Rev Panam Salud Publica. 2005;17(5-6):353-61.

8. American Geriatrics Society 2012 Beers Criteria Update Expert Panel. American Geriatrics Society updated Beers criteria for potentially inappropriate medication use in older adults. J Am Geriatr Soc. 2012;60(4):616-31. doi: 10.1111/j.1532-5415.2012.03923.x.

9. ResnickB,PacalaJT.2012Beerscriteria.J Am Geriatr Soc. 2012;60(4):612-3. doi: 10.1111/j.1532-5415.2012.03921.x.

10. Rosselli D, Ardila A, Pradilla G, Morillo L, Bautista L, Rey O, et al. El examen mental abreviado (Mini-Mental State Examination) como prueba de selección para el diagnóstico de demencia: estudio poblacional colombiano. Rev Neurol. 2000;30(5):428-32.

11. Gokula M, Holmes HM. Tools to reduce polypharmacy. Clin Geriat Med. 2012;28(2):323-41. doi: 10.1016/j. cger.2012.01.011.

12. Gnjidic D, Hilmer SN, Blyth FM, Naganathan V, Waite L, Seibel MJ, et al. Polypharmacy cutoff and outcomes: five or more medicines were used to identify community-dwelling older men at risk of different adverse outcomes. J Clin Epidemiol. 2012;65(9):989-95. doi: 10.1016/j.jclinepi.2012.02.018.

13. Haider SI, Johnell K, Weitoft GR, Thorslund M, Fastbom J. The influence of educational level on polypharmacy and inappropriate drug use: a registerbased study of more than 600,000 older people. J Am Geriatr Soc. 2009;57(1):62-9. doi: 10.1111/j.15325415.2008.02040.x.

14. Rojas M, Ruiz I, Carrasco VH, Molina JC. Caracterización de pacientes y de uso de medicamentos en un grupo de adultos mayores asistentes a una actividad promocional de geriatría. Rev Hosp Clín Univ Chile. 2008;19:31823.

15. Wise J. Polypharmacy: a necessary evil. BMJ. 2013;347:f7033. doi: 10.1136/ bmj.f7033.

16. Secoli SR, Figueras A, Lebrao ML, de Lima FD, Santos JL. Risk of potential drug-drug interactions among Brazilian elderly: a populationbased, cross-sectional study. Drugs Aging. 2010;27(9):759-70. doi: 10.2165/11538460-00000000000000.

17. Horne R, Weinman J. Patients' beliefs about prescribed medicines and their role in adherence to treatment in chronic physical illness. J Psychosom Res. 1999;47(6):555-67.

18. Vik SA, Maxwell CJ, Hogan DB. Measurement, correlates, and health outcomes of medication adherence among seniors. Ann Pharmacother. 2004;38(2):303-12.

19. Oscanoa TJ. [Safe use of medications among elderly people: a checklist].
Rev Peru Med Exp Salud Publica. 2013;30(2):320-5.

20. Wandel S, Juni P, Tendal B, Nuesch E, Villiger PM, Welton NJ, et al. Effects of glucosamine, chondroitin, or placebo in patients with osteoarthritis of hip or knee: network meta-analysis. BMJ. 2010;341:c4675. doi: 10.1136/bmj. c4675.

21. Lai SW, Liao KF, Liao CC, Muo CH, Liu CS, Sung FC. Polypharmacy correlates with increased risk for hip fracture in the elderly: a populationbased study. Medicine (Baltimore). 2010;89(5):295-9. doi: 10.1097/ MD.0b013e3181f15efc.

22. Marzi M M, Diruscio A V, Núñez H M, Pires S M, Quaglia B N. Análisis de la prescripción de medicamentos en una comunidad geriátrica Argentina. Rev Med Chil. 2013;141(2):194-201. doi: 10.4067/S0034-98872013000200008.

23. Bosboom PR, Alfonso $\mathrm{H}$, Almeida OP, Beer C. Use of potentially harmful medications and health-related quality of life among people with dementia living in residential aged care facilities. Dement Geriatr Cogn Dis Extra. 2012;2(1):361-71. doi: $10.1159 / 000342172$.

24. Ruggiero C, Dell'Aquila G, Gasperini B, Onder G, Lattanzio F, Volpato S, et al. Potentially inappropriate drug prescriptions and risk of hospitalization among older, Italian, nursing home residents: the ULISSE project. Drugs Aging. 2010;27(9):747-58. doi: 10.2165/11538240-00000000000000 .

25. Gallagher PF, O'Connor MN, O'Mahony D. Prevention of potentially inappropriate prescribing for elderly patients: a randomized controlled trial using STOPP/START criteria. Clinical Pharmacol Ther. 2011;89(6):84554. doi: 10.1038/clpt.2011.44.

Correspondencia: Diego Rosselli

Dirección: Carrera 7 No. 40 - 62, piso 2. Bogota, Colombia.

Teléfono: +5713208320 ext. 2808

Correo electrónico:diego.rosselli@gmail.com 